\title{
The Nonsmooth Vibration of a Relative Rotation System with Backlash and Dry Friction
}

\author{
Minjia He, ${ }^{1,2}$ Shuo Li, ${ }^{2}$ Jinjin Wang, ${ }^{1,2}$ Zhenjun Lin, $^{2}$ and Shuang Liu ${ }^{1,2}$ \\ ${ }^{1}$ Liren College of Yanshan University, Qinhuangdao 066004, China \\ ${ }^{2}$ College of Electrical Engineering, Yanshan University, Qinhuangdao 066004, China \\ Correspondence should be addressed to Shuo Li; shuoli@stumail.ysu.edu.cn
}

Received 2 May 2017; Accepted 14 August 2017; Published 7 November 2017

Academic Editor: Viktor Avrutin

Copyright (C) 2017 Minjia He et al. This is an open access article distributed under the Creative Commons Attribution License, which permits unrestricted use, distribution, and reproduction in any medium, provided the original work is properly cited.

\begin{abstract}
We investigate a relative rotation system with backlash and dry friction. Firstly, the corresponding nonsmooth characters are discussed by the differential inclusion theory, and the analytic conditions for stick and nonstick motions are developed to understand the motion switching mechanism. Based on such analytic conditions of motion switching, the influence of the maximal static friction torque and the driving torque on the stick motion is studied. Moreover, the sliding time bifurcation diagrams, duty cycle figures, time history diagrams, and the $K$-function time history diagram are also presented, which confirm the analytic results. The methodology presented in this paper can be applied to predictions of motions in nonsmooth dynamical systems.
\end{abstract}

\section{Introduction}

Relative rotation system is a widespread power transmission system, which contains many nonlinear factors such as the nonlinear damping, stiffness, and backlash. These nonlinear factors can generally lead to vibration, which will reduce the transmission efficiency and performance. Therefore, the vibration of the relative rotation system is always a hot research topic.

Since the theory of rotational relativistic mechanics was first established by Carmeli in 1985 [1,2], a lot of achievements on the relative rotation system have been obtained. Luo set up the theory of relativistic analytical mechanics of the rotational systems in [3, 4]. The Hopf bifurcation for a kind of nonlinear relative rotation system was investigated [5-7]. In [8,9], the bifurcation response equation of relative rotation system was deduced with the method of multiple scales, and the bifurcation and chaotic motions under combination resonance were investigated. The chaotic behaviors of a relative rotation nonlinear dynamical system under parametric excitation and its control were studied in $[10,11]$. The papers above made a better research on the vibration of the relative rotation system, but the nonsmooth factors were rarely taken into consideration.

At present, the nonsmooth dynamics are a hot research topic. One reason for this is that the nonsmooth system widely exists in different disciplines. Mechanical engineers study the stick-slip oscillations in systems with dry friction and the dynamics of impact phenomena with unilateral constraints. Electrical circuits contain diodes and transistors, which ideally behave in a nonsmooth way. Meanwhile, there have been a lot of researches on the nonsmooth dynamics. In [12], a research on a piecewise linear system without damping was made, and the closed-form solution for periodic response was obtained. In [13], a mapping technique was developed to investigate a linear system with a single discontinuity, and the chaotic behavior was presented numerically. In [14], a mapping approach was adopted to investigate the periodic response and bifurcation of a piecewise linear system. In [15], Luo initially proposed a mapping structure for discontinuous systems. In [16], the idea of mapping structure was used to investigate a periodic forced piecewise linear system. In addition, the investigations by Nordmark [17], Błazejczyk et al. [18], Blazejczyk-Okolewska et al. [19], Luo et al. [20], di Bernardo et al. [21, 22], and Luo et al. [23-26] can also provide us with a plenty of meaningful conclusions.

Among the various nonsmooth factors, dry friction extensively exists in engineering, such as disk brake systems, turbine blades, and string music instruments. The discontinuity caused by dry friction forces makes the system more difficult to solve theoretically and numerically. Therefore, the 


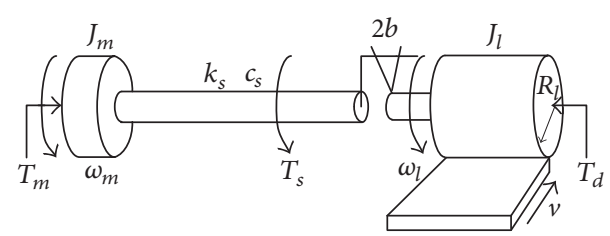

FIGURE 1: Mechanical model.

friction-induced oscillations have been of great interest for a long time. In [27], Hartog initially made an investigation on the periodic motion of the forced linear oscillator with Coulomb and viscous damping. In [28], from mathematical point of view, Levitan discussed a friction oscillation model, and the stability of the periodic motion was studied. In [29], Shaw studied the stability for a nonstick, periodic motion through the Poincare mapping. In [30], Feeny researched the nonsmoothness of a Coulomb friction oscillator and presented the stick region analytically and graphically. Feeny and Moon made the experimental and numerical investigations of chaos in a dry friction oscillator. In [31], Feeny and Moon investigated the dynamics of a oscillator with impact and friction. In [32, 33], the stick and nonstick motions were observed, and the chaos for a nonlinear friction model was presented. In [34], the theoretical approach was discussed to treat models of dynamical systems involving nonsmooth nonlinearities, which was associated with differential inclusions of mainly finite dimensional dynamical systems and the introduction of maximal monotone operators (graphs) in order to describe models of impact or friction.

In this paper, the nonsmooth vibration of a relative rotation system with backlash and dry friction is investigated, especially the sliding bifurcation caused by the dry friction. Moreover, the analytic conditions for stick and nonstick motions are developed, and the influence of the maximum static friction torque and the driving torque on the stick motion is predicted numerically.

\section{Mechanical Model}

The present paper investigates a relative rotation system with backlash and dry friction, and the nonlinear model is shown in Figure 1.

By Newton's theorem, the balance equations for the motor and load parts can be obtained as

$$
\begin{aligned}
J_{m} \ddot{\theta}_{m}+c_{m} \dot{\theta}_{m}+T_{s} & =T_{m} \\
J_{l} \ddot{\theta}_{l}+c_{l} \dot{\theta}_{l}+T_{d} & =T_{s}
\end{aligned}
$$

with

$$
\begin{aligned}
\theta_{d} & =\theta_{m}-\theta_{l}, \\
T_{s} & =k_{s} f\left(\theta_{d}\right)+c_{s} \dot{\theta}_{d},
\end{aligned}
$$

where $J_{m}$ is the motor moment of inertia, $J_{l}$ is the load moment of inertia, $T_{s}$ represents the transmitted shaft torque, $T_{m}$ is the motor torque, $T_{d}$ is the load torque disturbance, $c_{s}$ is the inner damping coefficient of the shaft, $c_{m}$ is the motor viscous friction coefficient, $c_{l}$ is the load viscous friction

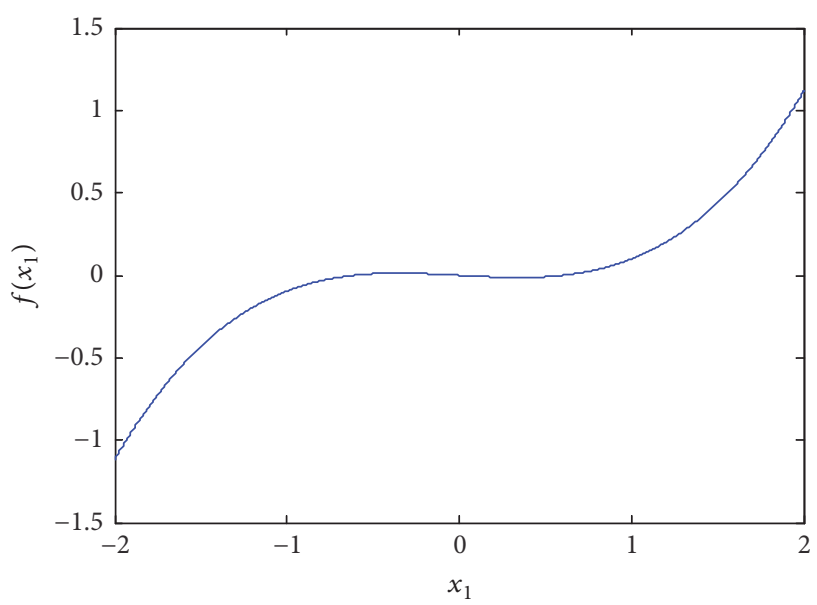

FIGURE 2: Approximate function.

coefficient, $k_{s}$ is the shaft elasticity, $2 b$ is the total backlash angle, and $f\left(\theta_{d}\right)$ is a dead zone function as

$$
f\left(\theta_{d}\right)= \begin{cases}\theta_{d}-b, & \theta_{d} \geq b \\ 0, & \left|\theta_{d}\right|<b \\ \theta_{d}+b, & \theta_{d} \leq-b .\end{cases}
$$

Let $x_{1}=\left(\theta_{m}-\theta_{l}\right) / b, x_{2}=\dot{\theta}_{m}$, and $x_{3}=\dot{\theta}_{l}$; we can deduce

$$
\begin{aligned}
\dot{x}_{1} & =\frac{1}{b} *\left(\dot{\theta}_{m}-\dot{\theta}_{l}\right)=\frac{1}{b} *\left(x_{2}-x_{3}\right) \\
\dot{x}_{2} & =\ddot{\theta}_{m}=\frac{1}{J_{m}} *\left(T_{m}-T_{s}-c_{m} \dot{\theta}_{m}\right) \\
& =\frac{1}{J_{m}} *\left[T_{m}-k_{s} b f\left(x_{1}\right)-c_{s}\left(x_{2}-x_{3}\right)-c_{m} x_{2}\right] \\
\dot{x}_{3} & =\ddot{\theta}_{l}=\frac{1}{J_{l}} *\left(T_{s}-T_{d}-c_{l} \dot{\theta}_{l}\right) \\
& =\frac{1}{J_{l}} *\left[k_{s} b f\left(x_{1}\right)+c_{s}\left(x_{2}-x_{3}\right)-T_{d}-c_{l} x_{3}\right]
\end{aligned}
$$

with

$$
f\left(x_{1}\right)= \begin{cases}x_{1}-1 & x_{1} \geq 1 \\ 0 & \left|x_{1}\right|<1 \\ x_{1}+1 & x_{1} \leq-1\end{cases}
$$

Suppose $\alpha=1 / b, k_{1}=k_{s} b / J_{m}, c_{1}=c_{s} b / J_{m}, c_{2}=c_{m} / J_{m}$, $c_{3}=c_{s} b / J_{l}, c_{4}=c_{l} / J_{l}, k_{2}=k_{s} b / J_{l}$, and $F_{f}=T_{d} / J_{l}$; then

$$
\begin{aligned}
& \dot{x}_{1}=\alpha\left(x_{2}-x_{3}\right) \\
& \dot{x}_{2}=-k_{1} f\left(x_{1}\right)-\left(c_{1}+c_{2}\right) x_{2}+c_{1} x_{3}+F \\
& \dot{x}_{3}=k_{2} f\left(x_{1}\right)+c_{3} x_{2}-\left(c_{3}+c_{4}\right) x_{3}-F_{f} .
\end{aligned}
$$

As for the dead zone function $f\left(x_{1}\right)$, it can be approximately expressed as $f\left(x_{1}\right)=0.1538 x_{1}{ }^{3}-0.0566 x_{1}$, which is shown in Figure 2.

For the system model (6) above, suppose the driving torque as $F=f_{0}+f_{1} \sin (\omega t)$ and the dry friction load torque as $F_{f}=f_{s} \operatorname{sign}\left(v_{l}-v\right)-\mu_{1}\left(v_{l}-v\right)+\mu_{2}\left(v_{l}-v\right)^{3}$, where 


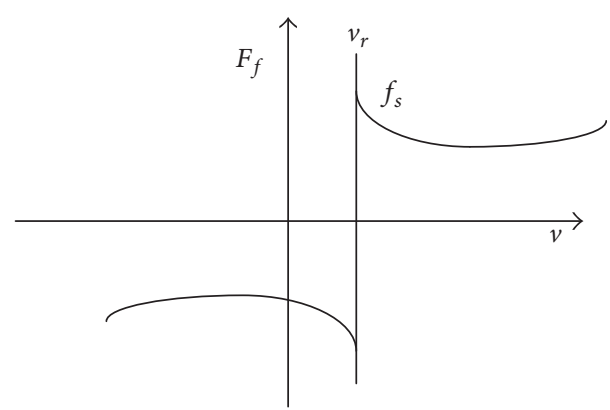

Figure 3: Dry friction.

$f_{s}$ is the maximal static friction torque, $\mu_{1}$ is the mixed friction coefficient, $\mu_{2}$ is the dynamic pressure friction coefficient, $v_{l}$ represents the line velocity of the load parts, $v_{l}=\dot{\theta}_{l} R_{l}$, and $v$ is the load velocity; therefore $F_{f}=f_{s} \operatorname{sign}\left(R_{l} x_{3}-v\right)-$ $\mu_{1}\left(R_{l} x_{3}-v\right)+\mu_{2}\left(R_{l} x_{3}-v\right)^{3}$, which can be approximately described as shown in Figure 3. Then, the system model is obtained as

$$
\begin{aligned}
\dot{x}_{1} & =\alpha\left(x_{2}-x_{3}\right) \\
\dot{x}_{2} & =-k_{1}\left(0.1538 x_{1}^{3}-0.0566 x_{1}\right)-\left(c_{1}+c_{2}\right) x_{2}+c_{1} x_{3} \\
& +f_{0}+f_{1} \sin (\omega t) \\
\dot{x}_{3} & =k_{2}\left(0.1538 x_{1}^{3}-0.0566 x_{1}\right)+c_{3} x_{2}-\left(c_{3}+c_{4}\right) x_{3} \\
- & f_{s} \operatorname{sign}\left(R_{l} x_{3}-v\right)+\mu_{1}\left(R_{l} x_{3}-v\right)-\mu_{2}\left(R_{l} x_{3}-v\right)^{3} .
\end{aligned}
$$

\section{Nonsmooth Analysis}

The dry friction load makes the relative rotation system a classical nonsmooth system, and the dynamics are obviously different from the smooth system.

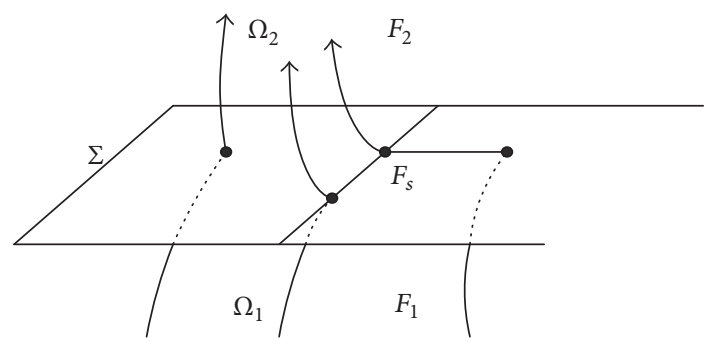

FIgURE 4: System domain.

For the system model in this paper, when the velocities of the load parts and the friction load are different, the friction size and direction are both changeable. The load friction direction will be opposite for the relative velocity larger and smaller than zero. Moreover, when the relative velocity is zero, the friction torque is uncertain. Therefore, this mechanical system model is a classical Filippov system, and we cannot deal with it using the conventional method.

In order to better analyze the nonsmooth dynamics, define the separation boundary as $\Sigma=\left\{x \in R^{3} \mid x_{3}-v / R_{l}=\right.$ $0\}$; then the system domain will be divided into three parts as

$$
\begin{aligned}
& \Omega_{1}=\left\{x \in R^{3} \mid x_{3}-\frac{v}{R_{l}}<0\right\} \\
& \Omega_{2}=\left\{x \in R^{3} \mid x_{3}-\frac{v}{R_{l}}>0\right\}
\end{aligned}
$$

and $\Sigma=\left\{x \in R^{3} \mid x_{3}-v / R_{l}=0\right\}$; they are shown in Figure 4 .

According to the discussion above, in the subregions $\Omega_{1}$ and $\Omega_{2}$, the equations of motion are as follows, respectively:

$$
\begin{aligned}
F_{1}(x) & =\left\{\begin{array}{l}
\dot{x}_{1}=\alpha\left(x_{2}-x_{3}\right) \\
\dot{x}_{2}=-k_{1}\left(0.1538 x_{1}^{3}-0.0566 x_{1}\right)-\left(c_{1}+c_{2}\right) x_{2}+c_{1} x_{3}+f_{0}+f_{1} \sin (\omega t) \\
\dot{x}_{3}=k_{2}\left(0.1538 x_{1}^{3}-0.0566 x_{1}\right)+c_{3} x_{2}-\left(c_{3}+c_{4}\right) x_{3}+f_{s}+\mu_{1}\left(R_{l} x_{3}-v\right)-\mu_{2}\left(R_{l} x_{3}-v\right)^{3},
\end{array}\right. \\
F_{2}(x) & =\left\{\begin{array}{l}
\dot{x}_{1}=\alpha\left(x_{2}-x_{3}\right) \\
\dot{x}_{2}=-k_{1}\left(0.1538 x_{1}^{3}-0.0566 x_{1}\right)-\left(c_{1}+c_{2}\right) x_{2}+c_{1} x_{3}+f_{0}+f_{1} \sin (\omega t) \\
\dot{x}_{3}=k_{2}\left(0.1538 x_{1}^{3}-0.0566 x_{1}\right)+c_{3} x_{2}-\left(c_{3}+c_{4}\right) x_{3}-f_{s}+\mu_{1}\left(R_{l} x_{3}-v\right)-\mu_{2}\left(R_{l} x_{3}-v\right)^{3} .
\end{array}\right.
\end{aligned}
$$

However, when the system flow arrives at the separation boundary, $F_{f}$ is not a determined value but a range as $F_{f} \in\left[-f_{s}, f_{s}\right]$; we cannot obtain the corresponding equation of motion. Therefore, the differential inclusion theory is introduced in this section to deal with it.

From the analysis above, the vector field can be described by a set-valued vector field as

$$
\dot{x} \in F(x)= \begin{cases}F_{1}(x) & x \in \Omega_{1} \\ \overline{c o}\left\{F_{1}(x), F_{2}(x)\right\} & x \in \Sigma \\ F_{2}(x) & x \in \Omega_{2},\end{cases}
$$

where $\overline{c o}\left\{F_{1}(x), F_{2}(x)\right\}$ is a vector field along the separation boundary, denoted by $F_{s}(x)$. From the convexity of the setvalued vector field, we have

$$
F_{s}(x)=\lambda F_{2}(x)+(1-\lambda) F_{1}(x),
$$

where $\lambda \in[0,1]$; then the system vector field can be described by a differential inclusion as follows:

$$
\dot{x} \in F(x)= \begin{cases}F_{1}(x) & x \in \Omega_{1}, \quad \lambda=0 \\ F_{s}(x) & x \in \Sigma, \quad \exists \lambda \in(0,1) \\ F_{2}(x) & x \in \Omega_{2}, \quad \lambda=1 .\end{cases}
$$




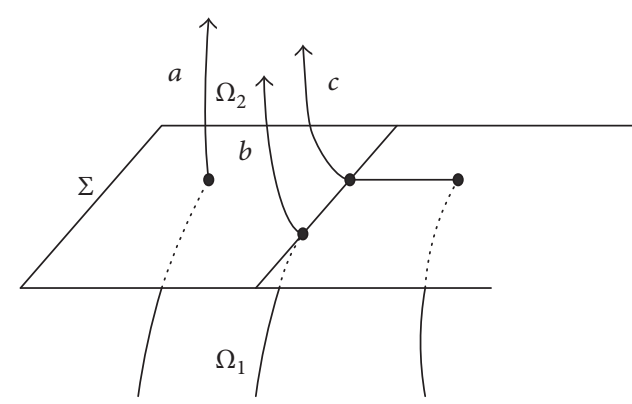

(a)

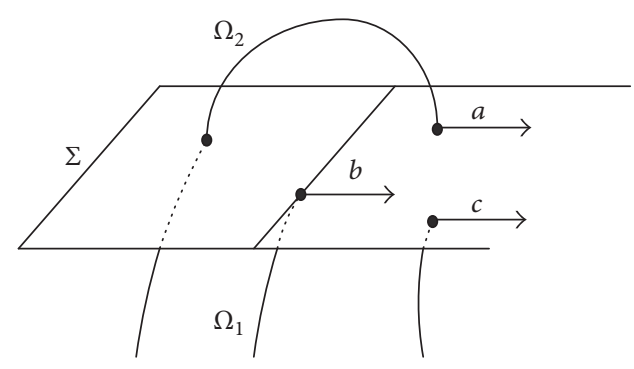

(c)

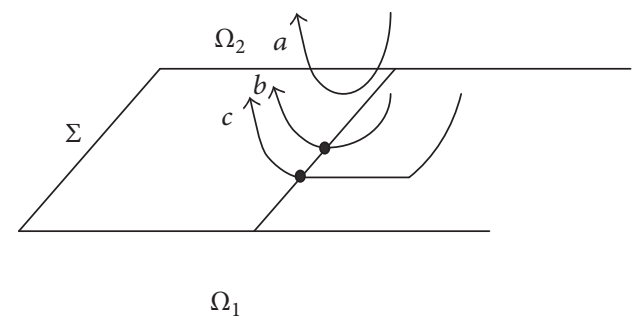

(b)

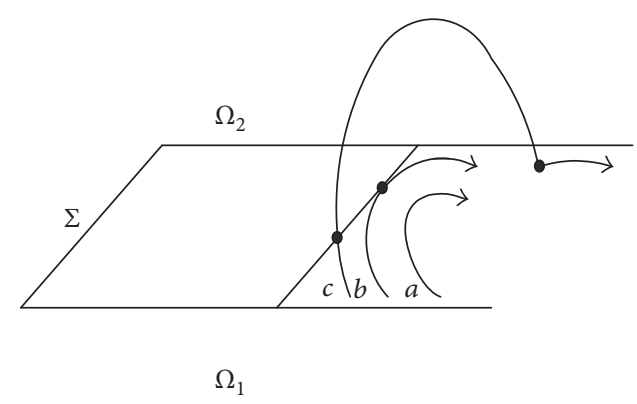

(d)

FIGURE 5: Sliding bifurcation type.

Since the vector field $F_{s}(x)$ determines the system flow along the separation boundary, we have $n_{\Sigma}^{T} F_{s}(x)=0$ with $n_{\Sigma}^{T}=$ $(0,0,1)$; then we get

$$
\lambda=\frac{n_{\Sigma}^{T} F_{1}(x)}{n_{\Sigma}^{T}\left[F_{1}(x)-F_{2}(x)\right]} .
$$

By using (11) and (13), the vector field $F_{s}(x)$ is obtained as

$$
F_{s}(x)=\left\{\begin{array}{l}
\dot{x}_{1}=\alpha\left(x_{2}-x_{3}\right) \\
\dot{x}_{2}=-k_{1}\left(0.1538 x_{1}^{3}-0.0566 x_{1}\right)-\left(c_{1}+c_{2}\right) x_{2}+c_{1} x_{3}+f_{0}+f_{1} \sin (\omega t) \\
\dot{x}_{3}=0 .
\end{array}\right.
$$

Then we can investigate the sliding dynamics along the separation boundary.

\section{Sliding Bifurcation}

The model in the present work is a classical Filippov system, and its typical nonsmooth phenomenon is the sliding bifurcation. According to the existing literatures, the sling bifurcation can be mainly divided into four types as follows.

Figure 5(a) depicts the scenario we term as sliding bifurcation of type I.

In the case presented in Figure 5(b), instead, a section of trajectory lying in region $\Omega_{1}$ or $\Omega_{2}$ grazes the boundary of the sliding region from above (or below). Again, this causes the formation of a section of sliding motion which locally tends to leave $\Sigma$. We term this transition as a grazing-sliding bifurcation.

A different bifurcation event, which we shall call sliding bifurcation of type II or switching-sliding, is depicted in
Figure 5(c). This scenario is similar to the sliding bifurcation of type I shown in Figure 5(a).

The fourth and last case is the so-called multisliding bifurcation, shown in Figure 5(d). It differs from the scenarios presented above, since the segment of the trajectory which undergoes the bifurcation lies entirely within the sliding region $\widehat{\Sigma}$.

Under the influence of the dry friction, the system flow may enter different subregions, and when the flow arrived at the separation boundary, it may stay on the boundary; then some type of sliding bifurcation may occur. Otherwise the system flow leaves the boundary. As for the different cases above, we give the corresponding analytic conditions in the following.

When the following equation is satisfied

$$
\left(n_{\Sigma}^{T} F_{1}(x)\right)\left(n_{\Sigma}^{T} F_{2}(x)\right)>0 \quad x \in \Sigma .
$$

the system flow will cross the separation boundary $\Sigma$. Meanwhile, when $n_{\Sigma}^{T} F_{1}(x)<0, n_{\Sigma}^{T} F_{2}(x)<0$, the system flow will 
enter the subregion $\Omega_{1}$, and when $n_{\Sigma}^{T} F_{1}(x)>0, n_{\Sigma}^{T} F_{2}(x)>0$, the system flow will enter the subregion $\Omega_{2}$.

When the following condition is satisfied

$$
\begin{aligned}
& n_{\Sigma}^{T} F_{1}(x)>0 \\
& n_{\Sigma}^{T} F_{2}(x)<0 \\
& \quad x \in \Sigma .
\end{aligned}
$$

the system flow will be sliding on the separation boundary. The stick motion occurs.

As for the sliding region, it is defined as

$$
\widehat{\Sigma}=\{x \in \Sigma: 0 \leq \lambda(x) \leq 1\}
$$

with

$$
\partial \widehat{\Sigma}=\partial \widehat{\Sigma}^{+} \cap \partial \widehat{\Sigma}^{-},
$$

where $\partial \widehat{\Sigma}^{-}=\{x \in \Sigma: \lambda(x)=0\}, \partial \widehat{\Sigma}^{+}=\{x \in \Sigma: \lambda(x)=1\}$. When $\lambda(x)=0$, we have $F_{s}(x)=F_{1}(x)$ and $n_{\Sigma}^{T} F_{1}(x)=0$; when $\lambda(x)=1$, we have $F_{s}(x)=F_{2}(x), n_{\Sigma}^{T} F_{2}(x)=0$. As for the stick motion, it may end in two cases as follows.

Case 1. The system flow leaves the separation boundary and enters the subregion $\Omega_{1}$.

Case 2. The system flow leaves the separation boundary and enters the subregion $\Omega_{2}$.

For the first case, it should satisfy the following:

$$
\begin{aligned}
& n_{\Sigma}^{T} F_{1}(x)=0 \\
& n_{\Sigma}^{T} F_{2}(x)<0
\end{aligned}
$$

$x \in \Sigma$

and for the second case, it should satisfy the following:

$$
\begin{aligned}
& n_{\Sigma}^{T} F_{1}(x)>0 \\
& n_{\Sigma}^{T} F_{2}(x)=0 \\
& x \in \Sigma .
\end{aligned}
$$

Then, by using the analytic conditions listed above, we can predict the sliding bifurcation for our system, which is presented in the next section.

\section{Numerical Predictions}

In this section, we investigate the sliding dynamics by using the analytic conditions listed above. Firstly, select the system parameters as

$$
\begin{aligned}
\alpha & =10, \\
k_{1} & =10, \\
k_{2} & =10, \\
c_{1} & =0.2, \\
c_{2} & =0.1, \\
c_{3} & =0.2, \\
c_{4} & =0.2, \\
\mu_{1} & =0.1, \\
R_{l} & =1, \\
v & =10,
\end{aligned}
$$

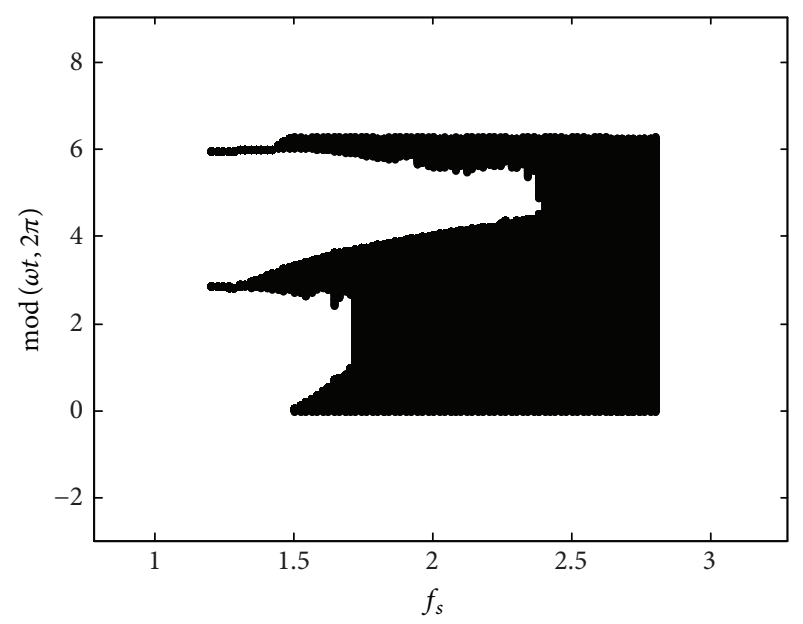

Figure 6: Sliding time bifurcation diagram.

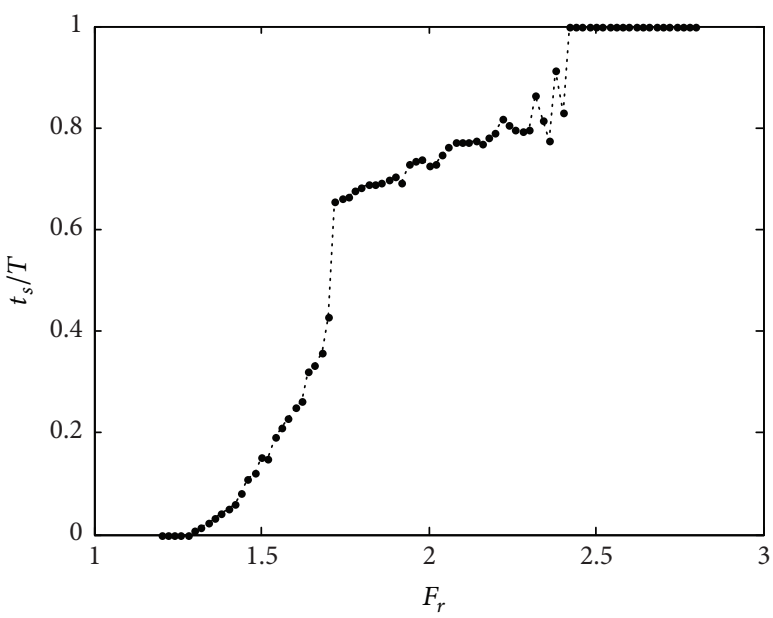

Figure 7: Sliding time duty cycle.

$$
\begin{aligned}
\mu_{2} & =0.0005, \\
f_{0} & =3, \\
f_{1} & =1.5, \\
\omega & =10 .
\end{aligned}
$$

Then, in order to better study the sliding dynamics, choose the separation boundary $\Sigma$ as the Poincare section. $\Pi=\left\{\left(x_{1}, x_{2}, x_{3}\right)^{T} \mid x_{3}-v / R_{l}=0\right\}$, and the Poincare mapping is as $\prod \rightarrow \prod$.

Choose the maximal static friction torque as the researched parameter; then we obtain the sliding bifurcation diagram and the sliding time duty cycle as shown in Figures 6 and 7.

From the bifurcation and the duty cycle diagram, we can conclude as follows: when the maximal static friction torque is smaller, there is no sliding bifurcation and the stick motion cannot be observed. As it increases, the sliding bifurcation occurs, and the sliding region shows the tendency of increase. When the static friction increased to a certain extent, the 

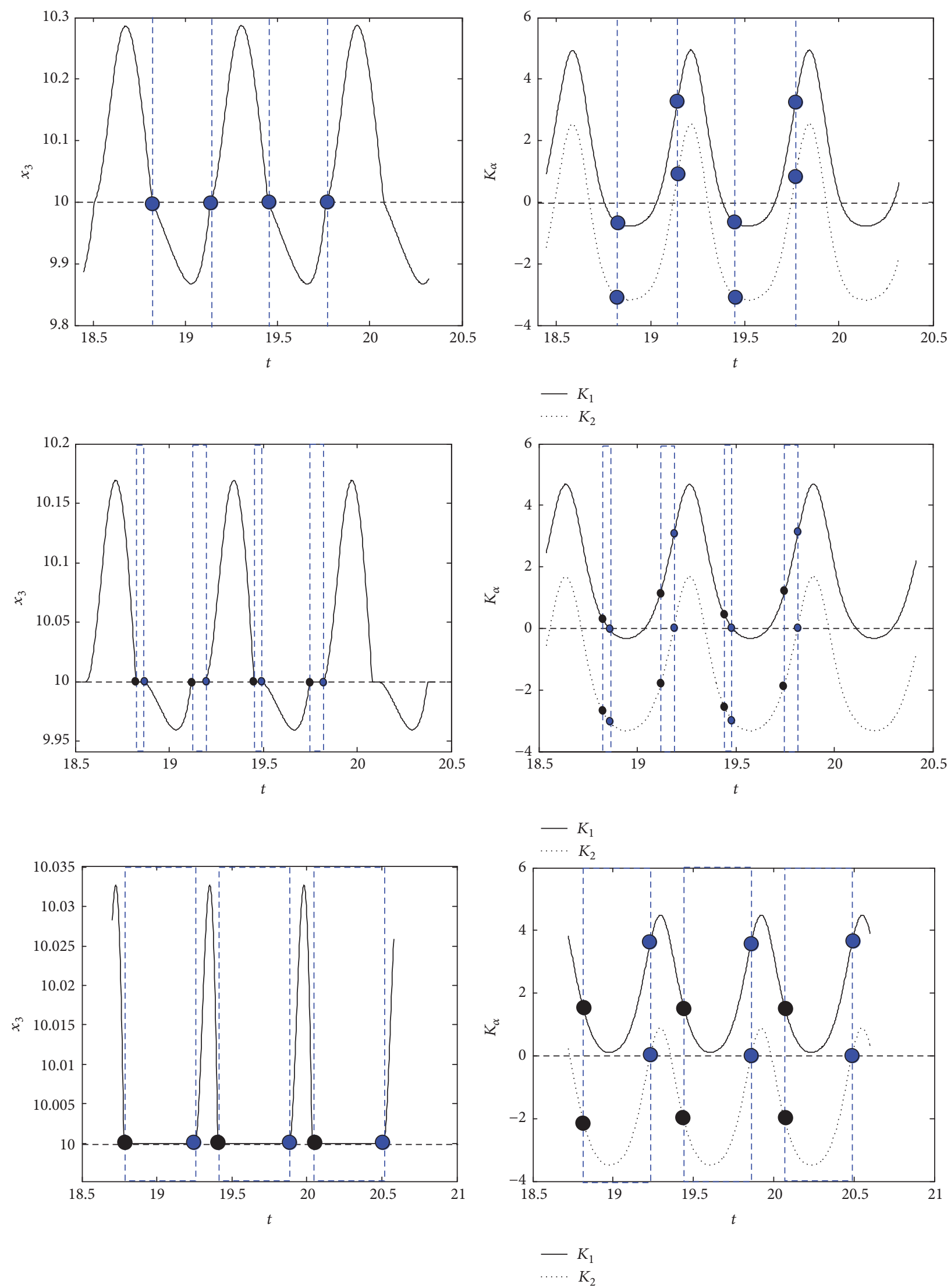

Figure 8: Continued. 

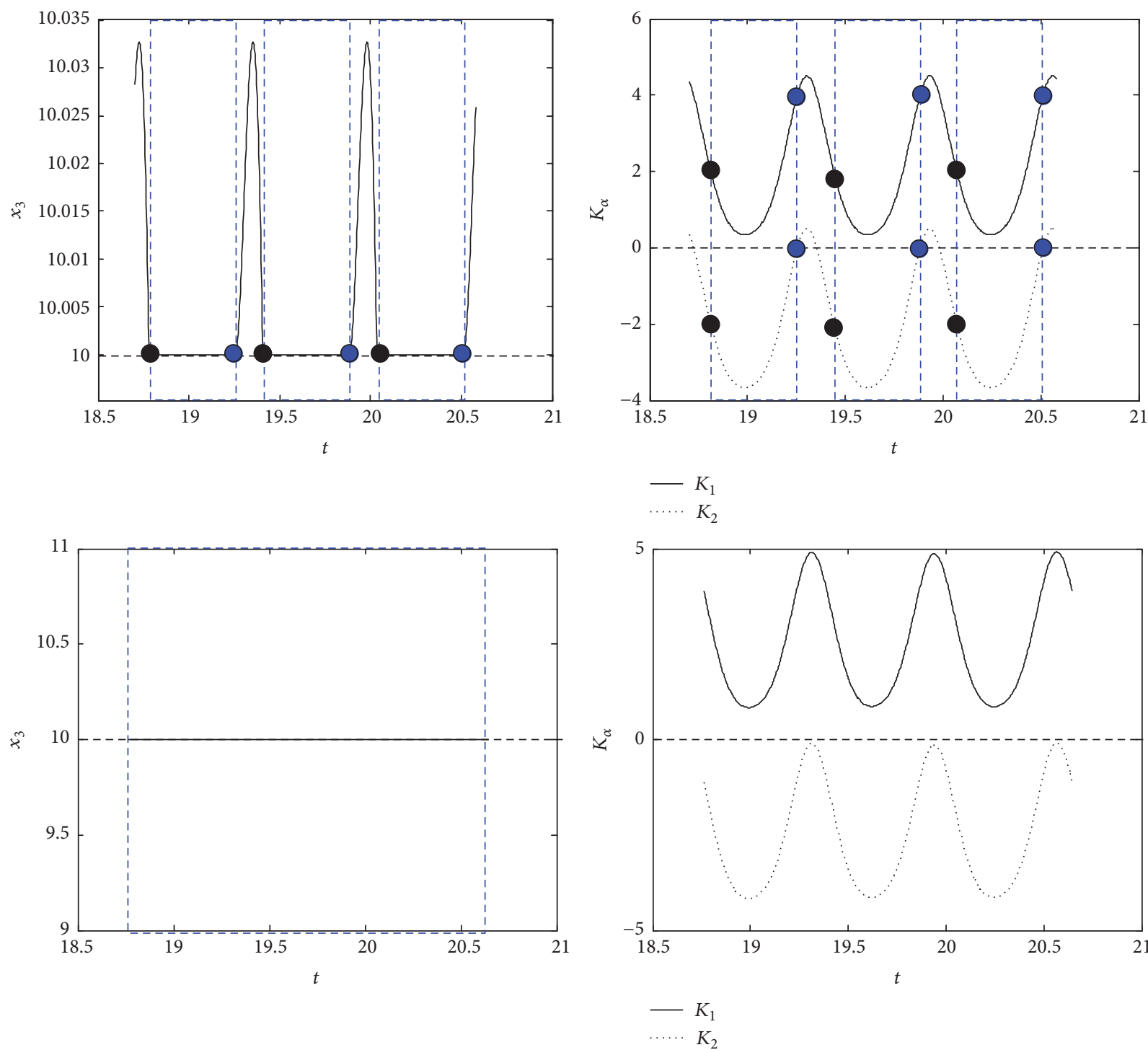

FIGURE 8: Time history and $K$-function diagram.

system flow always stayed on the separation boundary, which means that the stick motion occurs.

To better study the influence of maximal static friction torque on the sliding dynamics and verify the analytic conditions, define $K_{\alpha}=n_{\Sigma}^{T} F_{\alpha}(x), \alpha \in\{1,2\}$, and choose $f_{s}=$ $1.2,1.5,1.8,2.0,2.5$; then we can obtain the corresponding time history diagram and $K$-function diagram as shown in Figure 8.

From the above investigation, a conclusion is obtained as follows: when the maximal static friction torque is smaller $\left(f_{s}=1.2\right)$, the system flow crosses the separation boundary periodically; there exist no stick motions. As it increases, the sliding bifurcation occurs and the sliding region increases with the static friction torque increasing.

Next, we investigate the influence of the driving torque $f_{0}$ on the sliding dynamics. Choose the system parameters as $\alpha=10$,

$k_{1}=10$,

$k_{2}=10$,

$c_{1}=0.2$,

$c_{2}=0.1$,

$c_{3}=0.2$,

$c_{4}=0.2$,

$\mu_{1}=0.1$,

$R_{l}=1$,

$v=10$, 


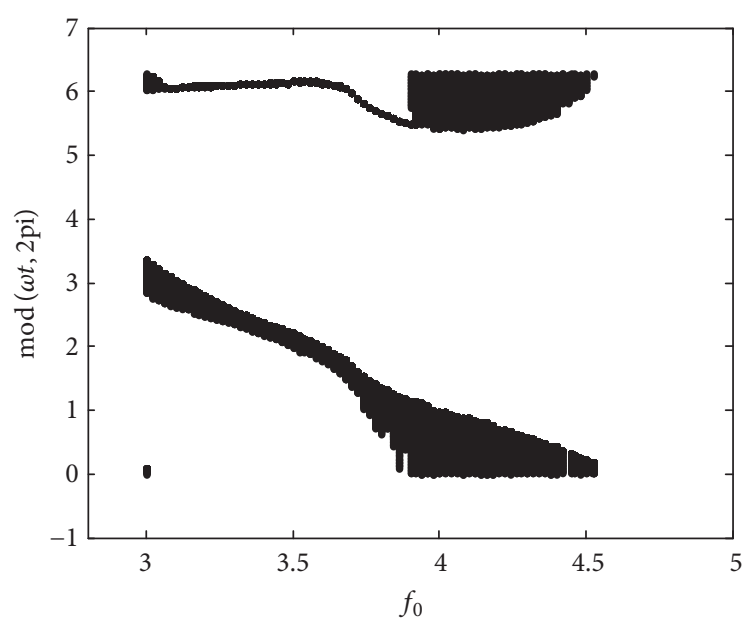

FIGURE 9: Sliding bifurcation.

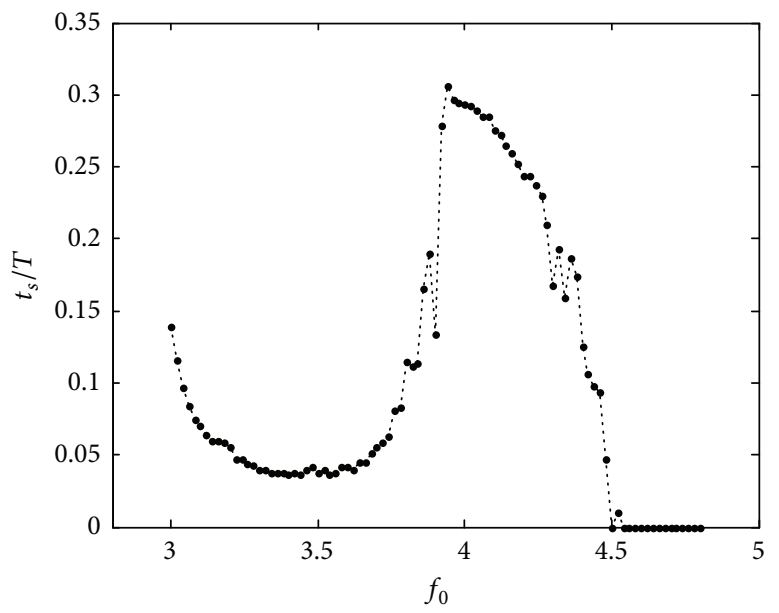

FIGURE 10: Sliding time duty cycle.

$\mu_{2}=0.0005$

$f_{1}=1.5$,

$f_{s}=1.5$,

$\omega=10$.

Then, we obtain the sliding bifurcation and the sliding time duty cycle as shown in Figures 9 and 10.

To better understand the influence of the driving torque $f_{0}$ on the sliding dynamics, select $f_{0}=3.5,4,4.5$ to make a detailed investigation; then the corresponding time history and $K$-function are shown in Figure 11.

By observing the numerical conclusions above, we can predict that the sliding region decreases with the increase of $f_{0}$ at first, then the sliding region increases, and at last the sliding region may disappear through the grazingsliding bifurcation. To verify the prediction, choose $f_{0}=$
$4.52,4.531,4.54$; then the corresponding dynamics are shown in Figure 12.

By observing Figure 12, when $f_{0}=4.532$, the grazingsliding bifurcation occurs; then the sliding region disappears, which is consistent with our prediction.

\section{Conclusion}

In this paper, we investigate the nonsmooth vibration of a relative rotation system with backlash and dry friction, especially the sliding bifurcation dynamics. The analytic conditions for the sliding dynamics are also obtained. Then, by using these conditions, we research the influence of the maximal static friction and the driving torque on the sliding dynamics. Moreover, the corresponding bifurcation diagram, duty cycle, time history diagram, and $K$-function are presented to predict the sliding bifurcation numerically, which are consistent with the analytic conditions.

The present work not only makes us have a deeper understanding of the sliding bifurcation dynamics, but also 

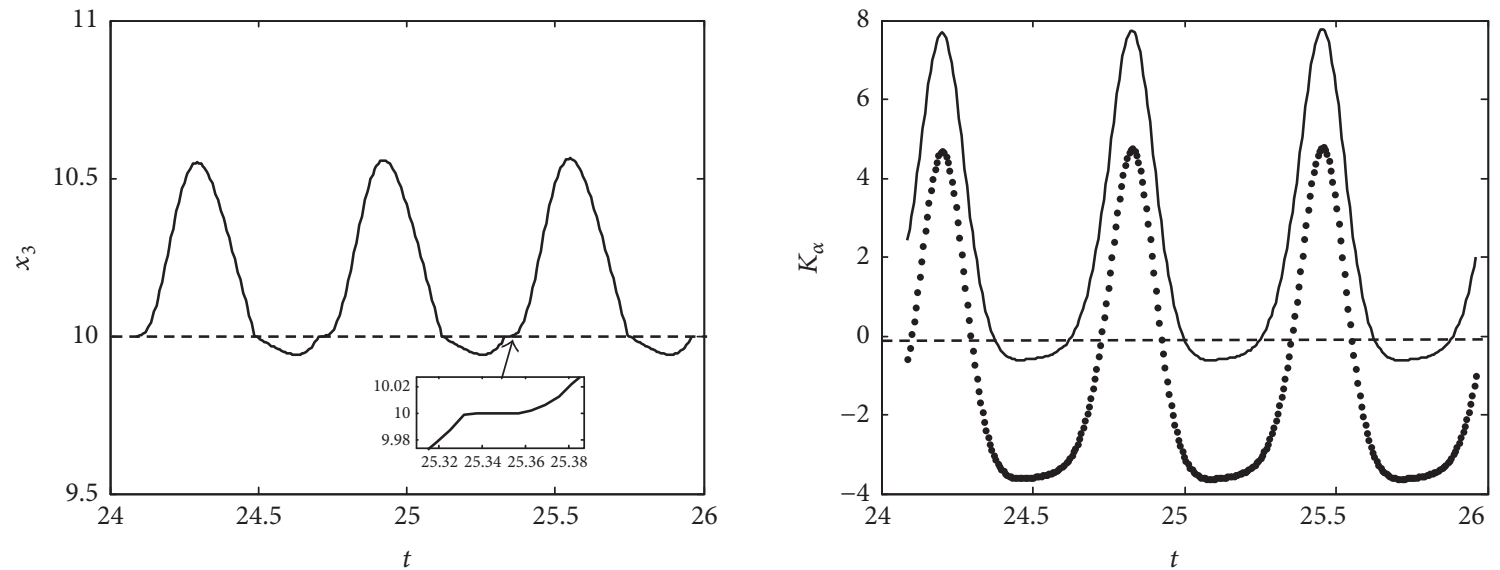

$K_{1}$
$-\quad K_{2}$
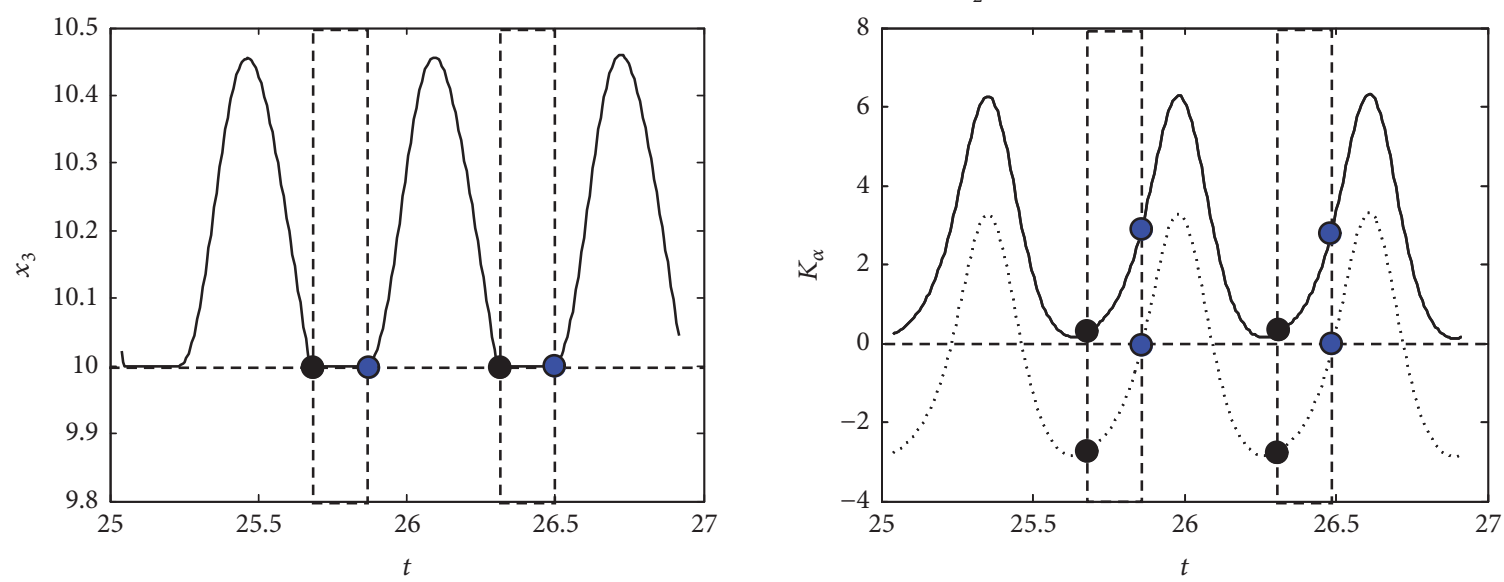

$\begin{array}{ll}- & K_{1} \\ \cdots \cdots & K_{2}\end{array}$
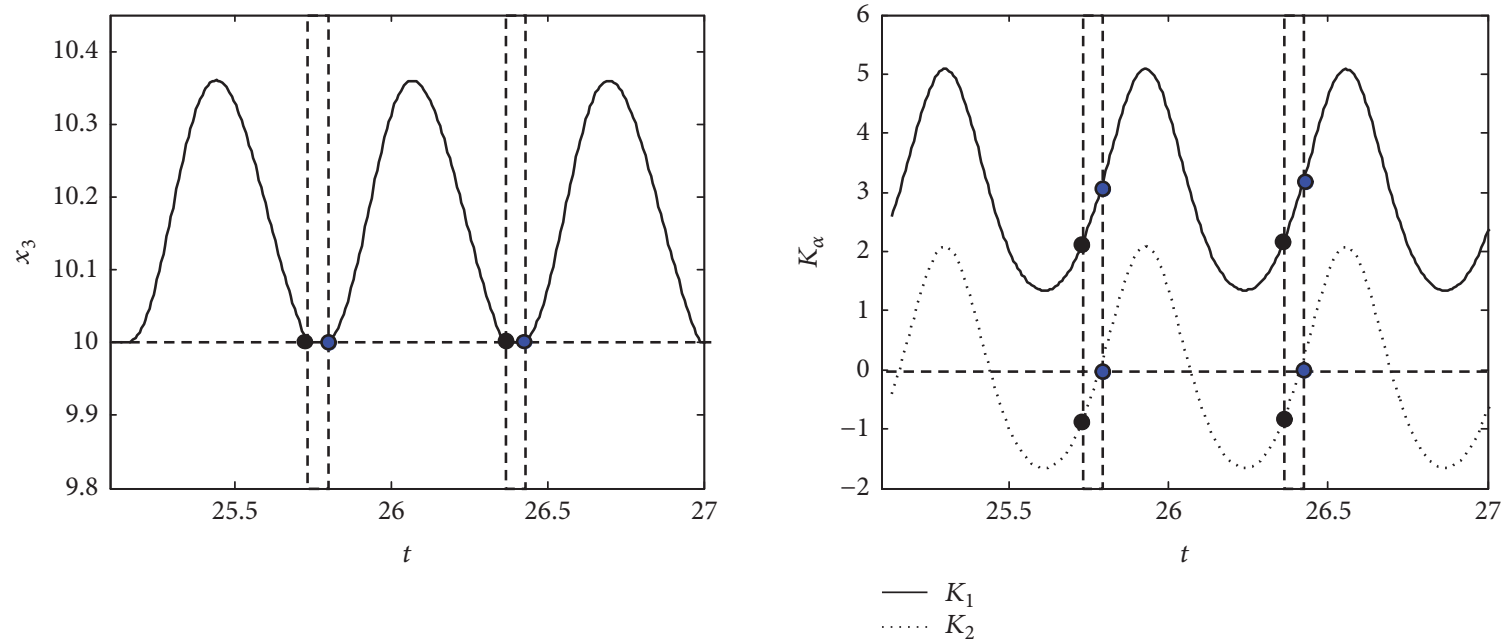

FIGURE 11: Time history and $K$-function. 

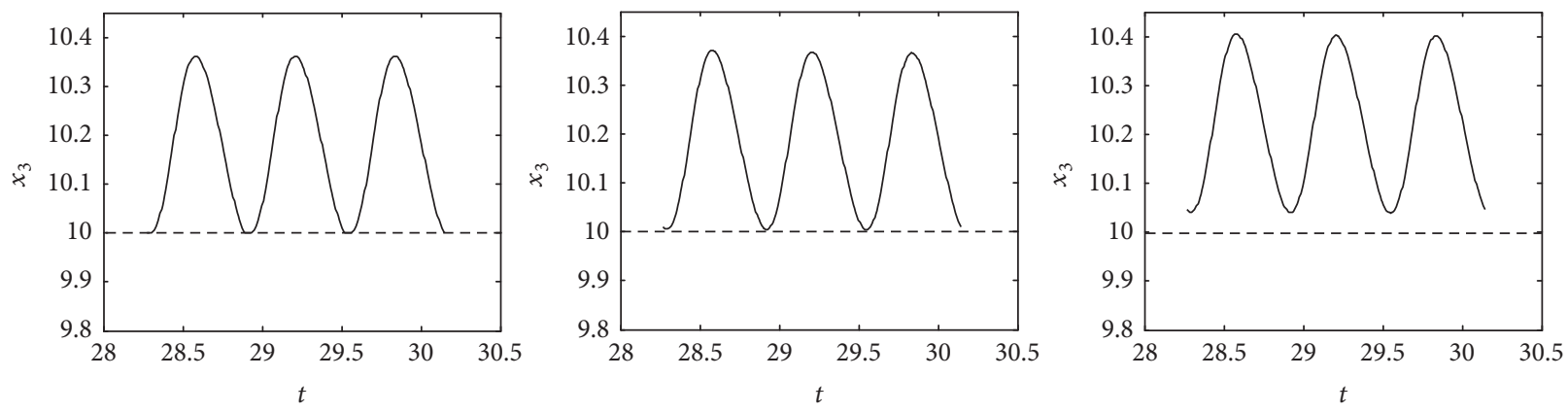

Figure 12: Time history diagram.

provides us with a method to study the nonsmooth vibration of the nonsmooth systems.

\section{Conflicts of Interest}

The authors declare that there are no conflicts of interest regarding the publication of this article.

\section{Acknowledgments}

The authors gratefully appreciate the support from National Natural Science Foundation of China (Grant no. 61673334).

\section{References}

[1] M. Carmeli, "Field theory on $R \times S^{3}$ topology. I. The KleinGordon and Schrödinger equations," Foundations of Physics. An International Journal Devoted to the Conceptual Bases and Fundamental Theories of Modern Physics, vol. 15, no. 2, pp. 175184, 1985.

[2] M. Carmeli, "Rotational relativity theory," International Journal of Theoretical Physics, vol. 25, no. 1, pp. 89-94, 1986.

[3] S. K. Luo, J. L. Fu, and X. W. Chen, "Basic theory of relativistic birkhoffian dynamics of rotational system," Acta Physica Sinica, vol. 50, no. 3, pp. 383-389, 2001.

[4] S. K. Luo, "The theory of relativistic analytical mechanics of the rotational systems," Applied Mathematics and Mechanics, vol. 19, no. 1, pp. 45-57, 1998.

[5] S. Liu, B. Liu, and P. M. Shi, "Nonlinear feedback control of Hopf bifurcation in a relative rotation dynamical system," Acta Physica Sinica, vol. 58, no. 7, pp. 4383-4389, 2009.

[6] B. Liu, Y. K. Zhang, S. Liu, and Y. Wen, "Hopf bifurcation and stability of periodic solutions in a nonlinear relative rotation dynamical system with time delay," Acta Physica Sinica, vol. 59, no. 1, pp. 38-43, 2010.

[7] S. Pei-Ming, L. Ji-Zhao, L. Bin, and H. Dong-Ying, "Stability and time-delayed feedback control of a relative-rotation nonlinear dynamical system under quasic-periodic parametric excitation," Acta Physica Sinica, vol. 60, no. 9, p. 094501, 2011.

[8] M. Zong, F. Li-Yuan, and S. Ming-Hou, "Bifurcation of a kind of nonlinear-relative rotational system with combined harmonic excitation," Acta Physica Sinica, vol. 62, no. 5, p. 054501, 2013.

[9] L. Hai-Bin, W. Bo-Hua, Z. Zhi-Qiang, L. Shuang, and L. YanShu, "Combination resonance bifurcations and chaos of some nonlinear relative rotation system," Acta Physica Sinica, vol. 61, no. 9, p. 094501, 2012.
[10] P. Shi, B. Liu, and D. Hou, "Global dynamic characteristic of nonlinear torsional vibration system under harmonically excitation," Chinese Journal of Mechanical Engineering, vol. 22, no. 1, pp. 132-139, 2009.

[11] P. M. Shi, D. Y. Han, and B. Liu, "Chaos and chaotic control in a relative rotation nonlinear dynamical system under parametric excitation," Chinese Physics B, vol. 19, no. 9, p. 090306, 2010.

[12] J. Hartog and S. Mikina, "Forced vibrations with non-linear spring constants," ASME Journal of Applied Mechanics, vol. 58, pp. 157-164, 1932.

[13] S. W. Shaw and P. J. Holmes, "A periodically forced piecewise linear oscillator," Journal of Sound and Vibration, vol. 90, no. 1, pp. 129-155, 1983.

[14] M. Kleczka, E. Kreuzer, and W. Schiehlen, "Local and global stability of a piecewise linear oscillator," Philosophical Transactions of the Royal Society of London. Series A. Mathematical, Physical Sciences and Engineering, vol. 338, no. 1651, pp. 533-546, 1992.

[15] A. C. J. Luo, Analytical modeling of bifurcation, chaos and multifractals in nonlinear dynamics, University of Manitoba, Manitoba, Canada, 1995.

[16] A. C. J. Luo and S. Menon, "Global chaos in a periodically forced, linear system with a dead-zone restoring force," Chaos, Solitons \& Fractals, vol. 19, no. 5, pp. 1189-1199, 2004.

[17] A. B. Nordmark, "Non-periodic motion caused by grazing incidence in an impact oscillator," Journal of Sound and Vibration, vol. 145, no. 2, pp. 279-297, 1991.

[18] B. Błazejczyk, T. Kapitaniak, J. Wojewoda, and R. Barron, "Experimental Observation Of Intermittent Chaos In A Mechanical System With Impacts," Journal of Sound and Vibration, vol. 178, no. 2, pp. 272-275, 1994.

[19] B. Blazejczyk-Okolewska, K. Czolczynski, and T. Kapitaniak, "Dynamics of a two-degree-of-freedom cantilever beam with impacts," Chaos, Solitons \& Fractals, vol. 40, no. 4, pp. 19912006, 2009.

[20] G. W. Luo, X. H. Lv, and Y. Q. Shi, "Vibro-impact dynamics of a two-degree-of freedom periodically-forced system with a clearance: diversity and parameter matching of periodic-impact motions," International Journal of Non-Linear Mechanics, vol. 65, no. 4, pp. 173-195, 2014.

[21] M. di Bernardo, C. J. Budd, and A. R. Champneys, "Normal form maps for grazing bifurcations in $\mathrm{n}$-dimensional piecewisesmooth dynamical systems," Physica D: Nonlinear Phenomena, vol. 160, no. 3-4, pp. 222-254, 2001.

[22] M. di Bernardo, A. Nordmark, and G. Olivar, "Discontinuityinduced bifurcations of equilibria in piecewise-smooth and impacting dynamical systems," Physica D: Nonlinear Phenomena, vol. 237, no. 1, pp. 119-136, 2008. 
[23] A. C. Luo, "A theory for non-smooth dynamic systems on the connectable domains," Communications in Nonlinear Science and Numerical Simulation, vol. 10, no. 1, pp. 1-55, 2005.

[24] C. Zhang, X. Han, and Q. Bi, "Dynamical behaviors of the periodic parameter-switching system," Nonlinear Dynamics, vol. 73, no. 1-2, pp. 29-37, 2013.

[25] A. C. Luo, "A periodically forced, piecewise linear system. I. Local singularity and grazing bifurcation," Communications in Nonlinear Science and Numerical Simulation, vol. 12, no. 3, pp. 379-396, 2007.

[26] A. C. J. Luo and L. Chen, "Periodic motions and grazing in a harmonically forced, piecewise, linear oscillator with impacts," Chaos, Solitons \& Fractals, vol. 24, no. 2, pp. 567-578, 2005.

[27] J. P. D. Hartog, "Forced vibrations with coulomb and viscous damping," Transactions of the American Society of Mechanical Engineers, vol. 53, pp. 107-115, 1931.

[28] E. S. Levitan, "Forced oscillation of a spring-mass system having combined Coulomb and viscous damping," The Journal of the Acoustical Society of America, vol. 32, pp. 1265-1269, 1960.

[29] S. W. Shaw, "On the dynamic response of a system with dry friction," Journal of Sound and Vibration, vol. 108, no. 2, pp. 305325, 1986.

[30] B. Feeny, "A nonsmooth Coulomb friction oscillator," Physica D: Nonlinear Phenomena, vol. 59, no. 1-3, pp. 25-38, 1992.

[31] B. Feeny and F. C. Moon, "Chaos in a Forced Dry-Friction Oscillator: Experiments and Numerical Modelling," Journal of Sound and Vibration, vol. 170, no. 3, pp. 303-323, 1994.

[32] N. Hinrichs, M. Oestreich, and K. Popp, "Dynamics of oscillators with impact and friction," Chaos, Solitons \& Fractals, vol. 8, no. 4, pp. 535-558, 1997.

[33] N. Hinrichs, M. Obstreich, and K. Popp, "On the modelling of friction oscillators," Journal of Sound and Vibration, vol. 216, no. 3, pp. 435-459, 1998.

[34] J. Bastien, F. Bernardin, C.-H. Lamarque, and N. Challamel, "Non-smooth Deterministic or Stochastic Discrete Dynamical Systems: Applications to Models with Friction or Impact," ISTE Ltd-Wiley, vol. 496, 2013. 


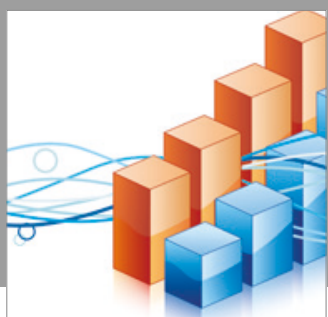

Advances in

Operations Research

vatersals

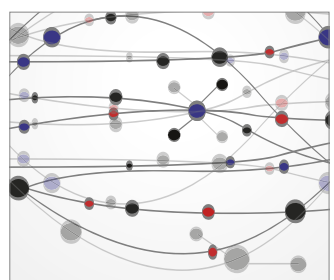

\section{The Scientific} World Journal
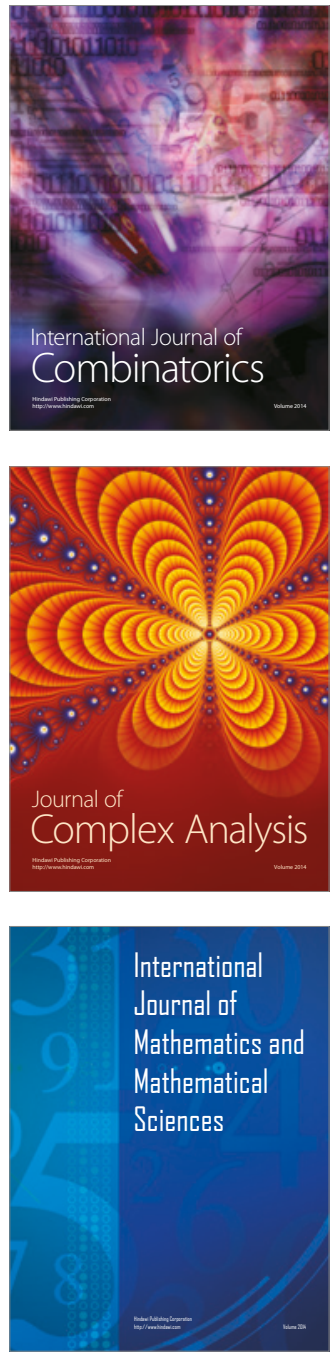
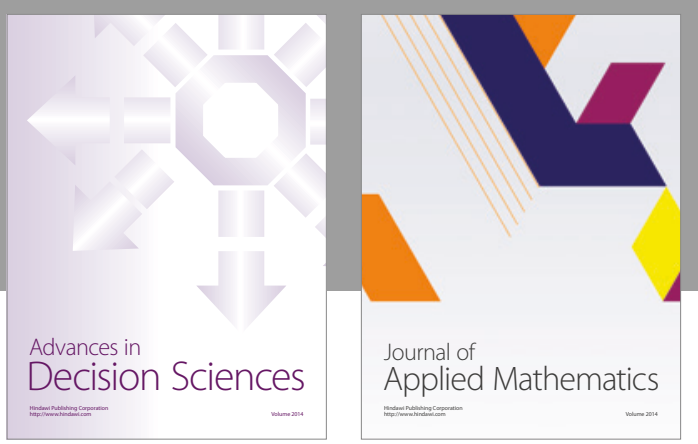

Algebra

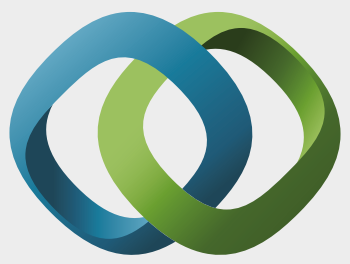

\section{Hindawi}

Submit your manuscripts at

https://www.hindawi.com
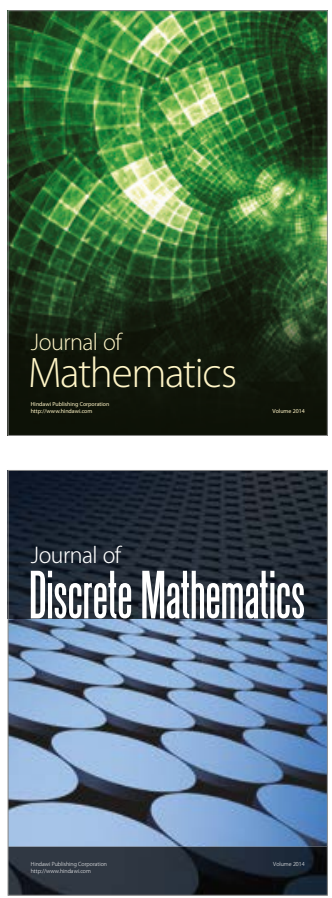

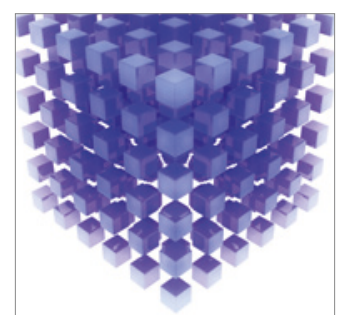

Mathematical Problems in Engineering
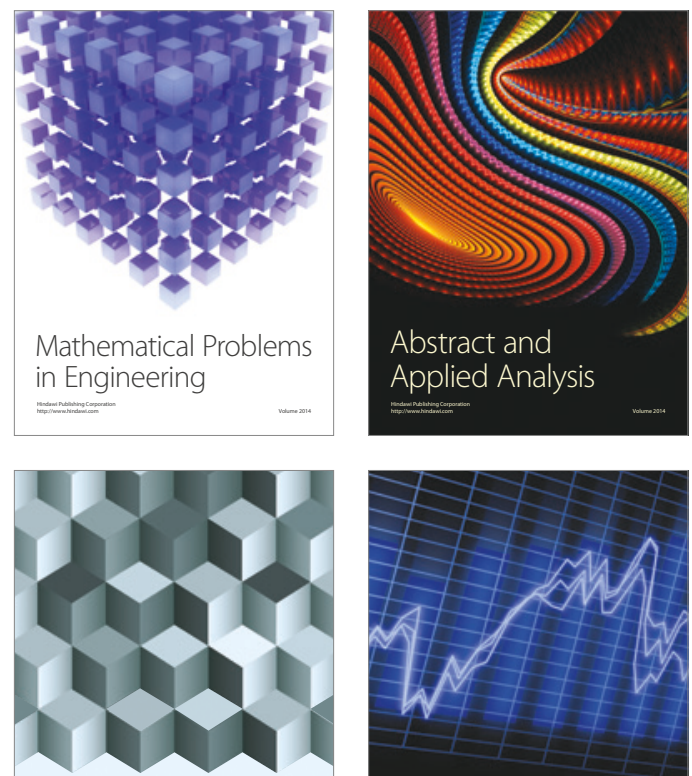

Journal of

Function Spaces

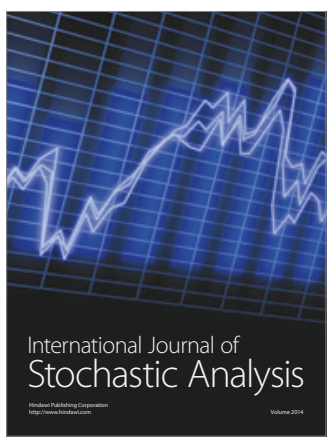

Probability and Statistics
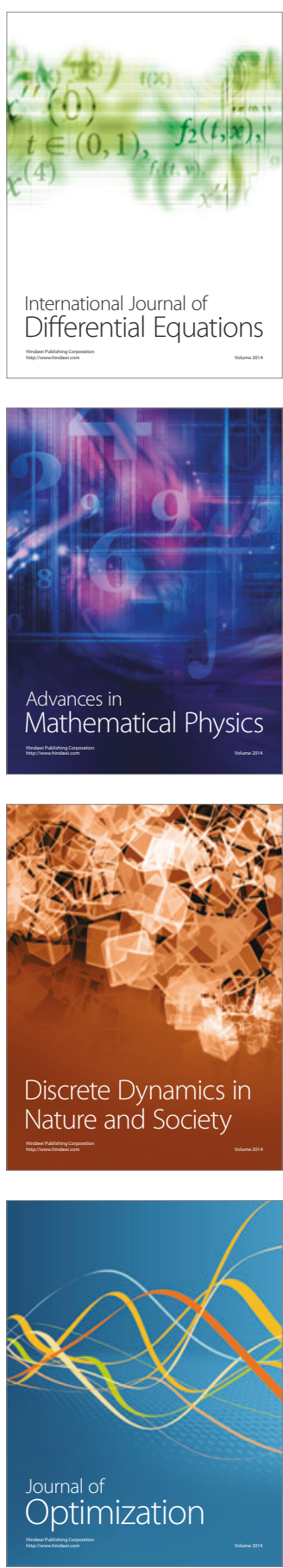